\title{
Node abnormality predicts seizure outcome and relates to long-term relapse after epilepsy surgery
}

Nishant Sinha ${ }^{1,2^{*}}$, Yujiang Wang ${ }^{1,2,3}$, Nádia Moreira da Silva ${ }^{1}$, Anna Miserocchi ${ }^{3}$, Andrew W. McEvoy ${ }^{3}$, Jane de Tisi ${ }^{3}$, Sjoerd B. Vos ${ }^{3,4,5}$, Gavin P. Winston ${ }^{3,5,6}$, John S. Duncan ${ }^{3,5}$, Peter Neal Taylor $1,2,3^{*}$

${ }^{1}$ CNNP Lab (www.cnnp-lab.com), Interdisciplinary Computing and Complex BioSystems Group, School of Computing, Newcastle University, Newcastle upon Tyne, United Kingdom

${ }^{2}$ Institute of Neuroscience, Faculty of Medical Sciences, Newcastle University, Newcastle upon Tyne, United Kingdom

${ }^{3}$ NIHR University College London Hospitals Biomedical Research Centre, UCL Institute of Neurology, Queen Square, London, United Kingdom

${ }^{4}$ Centre for Medical Image Computing, University College London, London, United Kingdom

${ }^{5}$ Epilepsy Society MRI Unit, Chalfont St Peter, United Kingdom

${ }^{6}$ Department of Medicine, Division of Neurology, Queen's University, Kingston, Canada

*Email: nishant.sinha89@gmail.com (NS); peter.taylor@newcastle.ac.uk (PNT) 


\section{Abstract}

Objective: We assessed pre-operative structural brain networks and clinical characteristics of patients with drug resistant temporal lobe epilepsy (TLE) to identify correlates of post-surgical seizure outcome at 1 year and seizure relapses up to 5 years.

Methods: We retrospectively examined data from 51 TLE patients who underwent anterior temporal lobe resection (ATLR) and 29 healthy controls. For each patient, using the pre-operative structural, diffusion, and post-operative structural MRI, we generated two networks: 'presurgery' network and 'surgically-spared' network. The pre-surgery network is the whole-brain network before surgery and the surgically-spared network is a subnetwork of the pre-surgery network which is expected to remain unaffected by surgery and hence present post-operatively. Standardising these networks with respect to controls, we determined the number of abnormal nodes before surgery and expected to remain after surgery. We incorporated these 2 abnormality measures and 13 commonly acquired clinical data from each patient in a robust machine learning framework to estimate patient-specific chances of seizures persisting after surgery.

Results: Patients with more abnormal nodes had lower chance of seizure freedom at 1 year and even if seizure free at 1 year, were more likely to relapse within five years. In the surgically-spared networks of poor outcome patients, the number of abnormal nodes was greater and their locations more widespread than in good outcome patients. We achieved $0.84 \pm 0.06$ AUC and $0.89 \pm 0.09$ specificity in detecting unsuccessful seizure outcomes at 1-year. Moreover, the 
model-predicted likelihood of seizure relapse was significantly correlated with the grade of surgical outcome at year-one and associated with relapses up-to five years post-surgery.

Conclusion: Node abnormality offers a personalised non-invasive marker, that can be combined with clinical data, to better estimate the chances of seizure freedom at 1 year, and subsequent relapse up to 5 years after ATLR.

\section{Introduction}

Epilepsy surgery is an effective treatment for bringing seizure remission in drug-resistant focal epilepsies, however, it is underutilised (Wiebe et al., 2001; Langfitt and Wiebe, 2008; Vakharia et al., 2018). One reason for the under-referral of patients is the reservations regarding the uncertainty of its outcome (Haneef et al., 2010; Vakharia et al., 2018). In around $30-40 \%$ of cases, seizures continue despite surgery and the multidisciplinary team are unable to accurately predict this risk prior to surgery (Janszky et al., 2005; Spencer and Huh, 2008; Téllez-Zenteno and Wiebe, 2008; de Tisi et al., 2011; Bell et al., 2017). Therefore, to better inform this clinical decision making, there is a need to predict seizure outcomes both in the short-term, and the likelihood of seizure relapse in the long term (Cohen-Gadol et al., 2008; de Tisi et al., 2011).

The incomplete removal of a wider epileptogenic network is increasingly being recognised as one of the reasons for continued seizures post-surgery (Spencer, 2002; Richardson, 2012). Many studies, driven by the aforementioned hypothesis, have attempted predicting seizure outcomes from presurgical data (Bonilha et al., 2015; Munsell et al., 2015; Goodfellow et al., 2016; Bell et al., 2017; Keller et al., 2017; Morgan et al., 2017; Proix et al., 2017; Sinha et al., 2017; Gleichgerrcht et al., 2018). Most 
studies, however, have investigated brain networks without incorporating the knowledge of the planned/performed surgery into the analysis. Naturally, the outcome of epilepsy surgery will depend not only on the pre-surgery brain network, but also on how the surgery (i.e., its location and extent) will affect the brain network (Taylor et al., 2018). Including surgical data allows the inference of a 'surgically-spared' network - the subnetwork for which none of the connections are altered by surgery and are therefore expected to remain after the surgery. Thus, the presence of epileptogenic structures in the surgically-spared network, a likely cause for seizure relapse in short or long term after surgery, needs investigation.

Studies employing quantitative imaging have consistently demonstrated that in temporal lobe epilepsy there are structural abnormalities that involve brain structures beyond the hippocampus and the temporal lobe (Bernasconi et al., 2004; Keller and Roberts, 2008; McDonald et al., 2008; Concha et al., 2012; Otte et al., 2012; Deleo et al., 2017). Accumulating evidence suggests that these abnormalities configure a network of abnormal structures that may be involved in the generation of seizures (Spencer, 2002; Bonilha et al., 2013; Bernhardt et al., 2015; Bonilha and Keller, 2015). Indeed, the pathophysiological mechanisms associated with epileptogenesis have a strong basis in aberrant neural connectivity (Liu et al., 2016; Besson et al., 2017). Therefore, quantifying the abnormalities before, and expected to remain after, surgery may inform postoperative seizure outcome.

The main goal of our study was to understand how structural network abnormality related to seizure outcomes after temporal lobe epilepsy surgery. We investigated the abnormality of the 'surgicallyspared' networks because, at a conceptual level, post-operative outcomes will likely be determined by what remains post-surgery. Our study addresses three main questions: a) do patients with more 
abnormalities have worse postoperative seizure outcomes? b) does surgery have a greater effect on node abnormality in seizure-free patients? c) if the node abnormality measure is to be used alongside common clinical variables of a patient, would it generalise to make patient-specific predictions on the chances of seizure freedom after surgery? Our study shows that the node abnormality is an important measure to be considered alongside other pre-surgical clinical factors to evaluate the risk of poorer seizure outcomes in patients with refractory TLE. 


\section{Methods}

\section{Participants}

We studied 51 patients who underwent unilateral anterior temporal lobe resection at the National Hospital of Neurology and Neurosurgery, London, United Kingdom and 29 healthy controls. Presurgical clinical information included: sex, age at epilepsy onset, age at surgery, epilepsy duration, number of AEDs taken before surgery, history of status epilepticus, history of secondary generalised seizures, side of surgery, evidence of MRI pathology, evidence of hippocampal sclerosis, history of depression, history of psychosis, and history of any other psychiatric disorders. Patients were followed up after surgery and classified according to the ILAE scale of seizure outcome at 12-month intervals (Wieser et al., 2001). One year after the surgery, 34 patients were completely seizure free (ILAE 1), 8 patients continued to have auras only (ILAE 2), and 9 patients were not seizure free (ILAE 3-6). This group of patients constitute the same cohort as in our previous study (Taylor et al., 2018).

ILAE surgical outcomes of seizure freedom were recorded annually at years 1 and 2 for all 51 patients, at year 3 for 45 patients, year 4 for 37 patients, and at year 5 for 31 patients. We considered that a patient had a seizure relapse if, at any given year after year 1 , the ILAE outcome of the patient changed from ILAE 1-2 to ILAE 3-6. If the ILAE outcome of a patient did not change to ILAE 3-6 and the followup duration was less than five years, it cannot be ascertained if the patient would have relapsed upon a full five-year follow-up. Therefore, beyond the known follow-up period, we did not include such patients in our analysis. The full preoperative clinical information, postoperative ILAE outcomes, and relapse data for each patient is provided in Table S1. 
We used $\chi^{2}$ test to check for the differences between the outcome groups in sex, side of surgery, evidence of hippocampal sclerosis, evidence of MRI pathology, history of status epilepticus, evidence of generalised seizures, history of depression, psychosis, and any other psychiatric disorders. Differences in age at epilepsy onset, age at surgery, epilepsy duration, and number of anti-epileptic drugs taken preoperatively between the outcome groups, were assessed with two-tailed nonparametric Wilcoxon ranksum test. Table 1 shows the summary of patient details.

\section{[Table 1]}

As a control group, we studied 29 healthy individuals, with no significant medical history of neurological or psychiatric problems. The control group was age and gender matched to the patient group. Control data was used as a normative measure for the metrics obtained from patients. The study was approved by the NHNN and the Institute of Neurology Joint Research Ethics Committee, and written informed consent was obtained from all subjects.

\section{MRI acquisition, data processing, and surgery network}

For each patient in this study, T1-weighted structural (sMRI) and diffusion-weighted (dMRI) data were acquired before the surgery. Within one year after the surgery, T1-weighted sMRI data was acquired again for each patient. Detailed imaging protocols are described in Supplementary Methods. Postoperative sMRI was used to accurately delineate the resected tissue. Complete details on how we drew the resection mask for these subjects are published in (Taylor et al., 2018). Briefly, the postoperative SMRI data was linearly registered to the preoperative SMRI using FSL, then the patient- 
specific resection masks were manually drawn, and finally validated by two independent raters for a majority subset.

Next, we applied the same data processing pipeline as in our previous study (Taylor et al., 2018) to incorporate the information of surgery for inferring the two networks: whole-brain pre-surgery network and surgically-spared subnetwork. Different processing steps involved in generating these networks are detailed in Supplementary Methods. In brief, the preoperative sMRI data were parcellated into 114 cortical and subcortical regions of interest (ROIs) derived from the predefined Geodesic Information Flow atlas and separately in 82 ROIs using the Freesurfer Desikan-Killiany atlas in the native space of each participant. We registered the parcellated ROls, resection mask, and tracts from deterministic tractography on $\mathrm{dMRI}$ data in native space. The pre-surgical streamline network is the connectivity matrix depicting the number of streamlines connecting two ROIs. The surgicallyspared streamline network is inferred after removing the streamlines that intersected the resection mask. By definition, surgery can only cause an immediate reduction in the number of streamlines. Therefore, we specified that the surgically-spared network contains only those network edges which are not expected to change in streamline count following surgery (i.e., edges where their streamlines do not pass through/into the resection cavity). These concepts are illustrated in Figure 1.

\section{[Figure 1]}

T1-weighted MRI and dMRI data were also acquired for 29 control participants using the same MRI scanner and imaging protocols as the patients. 


\section{Node abnormality computation}

The overall pipeline summarising different steps to compute node abnormality is illustrated in Figure

2. For each subject, we inferred networks based on the mean generalised fractional anisotropy (gFA) property of the streamlines obtained from the dMRI data (Tuch, 2004). We standardised the presurgery gFA network of each patient against controls as follows: for each connection present between ROIs $i$ and $j$ in a patient, the connection distribution was obtained from the equivalent connection between ROIs $i$ and $j$ from the control networks. The z-score for that connection was calculated as the number of standard deviations away from the mean, where the standard deviation and mean were obtained from the control distribution. Networks inferred from deterministic tractography are sparse, so we z-scored only those connections in patients for which an equivalent connection existed in at least 10 ( 35\%) controls (de Reus and van den Heuvel, 2013). This is depicted in Figure 2a.

\section{[Figure 2]}

After obtaining the pre-surgery z-scored gFA network, we removed the connections present in the surgery-affected network to obtain the surgically-spared z-transformed gFA network. High $|z|$ indicates high deviation from normality. Thus, the pre-surgery network maps the abnormal links present before the surgery and surgically-spared network maps the abnormal links that would remain unaffected immediately after the surgery. This is illustrated in Figure $2 b-d$.

To study how different regions (nodes) are affected in these networks, we computed node abnormalities (Figure 2e) in the pre-surgery and surgically-spared networks by counting the number of 
abnormal links to each node. We normalised the number of abnormal links to a node by its degree in the pre-surgical network, thus expressing node abnormality in percentage terms.

Quantification of node abnormality load raises two questions: first, what is the definition of an abnormal connection? second, when is a node considered abnormal? The former is essential for the application of a threshold on the abnormality network to count the number of abnormal links at each node. For the latter, another threshold is needed to define beyond what percentage level a node should be considered abnormal. We therefore varied the z-score threshold from 2.1 to 4.5 in increments of 0.1 and the percentage abnormality threshold from $1 \%$ to $50 \%$ in increments of $1 \%$. At each point on this two-dimensional grid, we computed how many nodes were abnormal in pre-surgical and surgically-spared networks. This is illustrated in Figure $2 \mathrm{f}$ for six example threshold pairs. Finally, having quantified the abnormality load for each patient, we assessed its discriminatory ability in predicting seizure outcomes and seizure relapse.

\section{Quantifying the change in abnormality load after ATLR}

To investigate the effect of surgical treatment on reduction of abnormalities, we compared the change in abnormality load between the pre-surgery and the surgically-spared networks. The ROls in the left and right hemispheres of patients were expressed as ipsilateral or contralateral to surgery. Then, we categorised each ROI into 6 ipsilateral and 6 contralateral areas i.e., temporal, subcortical, parietal, occipital, frontal, and cingulate cortices. In each area, we determined the number of abnormal nodes in the pre-surgery and surgically-spared networks patient-specifically. Then, we averaged the number of abnormal nodes in each area for the seizure free (ILAE 1) and non-seizure free (ILAE 3-6) groups of patients. Finally, by computing the proportion of abnormal nodes in every area (i.e., ratio of mean 
abnormal ROls to the total number of ROIs in each area) for the pre-surgery and surgically-spared network, we noted the change due to the surgery in all patients.

\section{Predictive model design for generalizability assessment}

We predicted the patient-specific probability of seizure relapse using preoperative clinical data, presurgery node abnormality, and the surgically-spared node abnormality. We performed this using support vector machine (SVM) implemented in MATLAB 'fitcsvm' classification library (Platt, 1999; Guyon et al., 2002). We applied a linear kernel because this enables the interpretation of weight vectors (i.e., the relative importance of each feature in the prediction), which were used to rank the importance of metrics in identifying patients who would have suboptimal seizure outcome. SVMs were initially trained with all 15 preoperative metrics: 13 clinical, 1 pre-surgery node abnormality, and 1 surgically-spared node abnormality. To identify the most informative metrics, after each round of SVM training, we removed the least important metric (in terms of its weight vector) and trained a new SVM with the remaining metrics. We repeated this process until only a single metric remained (Guyon et al., 2002; Fagerholm et al., 2015). At each stepwise removal we recorded: a) the performance of classifier in classifying totally seizure free (ILAE 1) and non-seizure free (ILAE 3-6) patients, and b) the Spearman's rank correlation between the predicted probability of seizure relapse for each patient with the actual severity of seizure outcomes at one-year after surgery (ILAE class).

The performance of the classifier was estimated using binary classification. Given that ILAE 2 patients tend to relapse (Table 1, S1), and thus, are in the spectrum between the totally seizure free (ILAE 1) and non-seizure free (ILAE 3-6) patients, we first excluded the ILAE outcome group 2 patients (Fairclough et al., 2018). With these patients removed, our dataset consisted of 43 samples, 34 of which 
were labelled 1 corresponding to ILAE 1 , and 9 were labelled -1 , corresponding to ILAE $3-6$. On this dataset, we performed nested-cross validation by combining a three-way split of the data (trainingvalidation-testing) with leave-one-out cross-validation (CV) and grid search for SVM parameter (boxconstraint) tuning. This was done to avoid upward bias in the metrics of performance estimates (Guyon and Elisseeff, 2003; Tsamardinos et al., 2018). Additionally, we avoided any bias in the selection of the most discriminatory threshold pair (i.e., z-score and percentage abnormality) to determine the node abnormality by computing it at every step of cross-validation after removing the test subject (Smialowski et al., 2009).

Specifically, in nested-cross validation, an external leave-one-out is implemented in which one patient is left out at every step for testing and the remaining patients used for training and validation. Training and validation were performed in the internal leave-one-out CV in which one patient is again left out for validation and the remaining used for model training combined with model parameter tuning. In our analysis, we tuned the model on 100 logarithmically spaced grid points between 1 and 10 . At every point, the SVM is trained and its performance tested using the patient left out for validation by estimating AUC. We selected the model parameter that gave the highest cross-validated AUC. The classifier generalisation capability is then evaluated by computing the classification AUC, accuracy, sensitivity, and specificity using the patient originally left for testing in the external cross-validation. We also noted the probability with which each test patient was classified as non-seizure free. The intuition being that the predictive model, though blind to the non-seizure free outcome categories (i.e., all ILAE 3 to ILAE 6 are labelled as -1), would classify the patients with worse surgical outcome with a higher probability. 
To determine where the ILAE outcome group 2 subjects fall on the spectrum, we treated all 8 ILAE outcome group 2 patients as test subjects. SVMs were trained and tuned, as described above, on all the remaining seizure free (ILAE 1) and non-seizure free (ILAE 3-6) patients (43 patients). On the classifier with highest discrimination between the seizure free and non-seizure free patients, we tested the features of ILAE 2 patients to note only the probability of classification to the non-seizure free group. We refer to these probabilities as the likelihood of seizure relapse because a high probability indicates a predicted propensity towards a non-seizure free outcome. Having obtained the likelihood of seizure relapse for all 51 patients, we compared this with the surgical outcome categories at year 1 and the actual seizure relapse in five years post-surgery. Note that the labels for all training data are binary and based on 12-month ILAE1 versus ILAE3-6 outcomes only. The model is therefore blind to severity of outcome (i.e. ILAE class $2,3,4,5$ ), and also blind to outcomes beyond 12 months.

\section{Statistical analysis and data availability}

To investigate if a greater number of abnormal nodes are associated with suboptimal seizure outcomes post-surgery, we applied the non-parametric Wilcoxon rank-sum test. One-tailed $p$ value was computed using the ranksum function in MATLAB incorporating the exact method. Effect size between groups was computed using Cohen's $d$ score, and the correlation coefficients between likelihood of seizure relapse and the severity of seizure outcome were determined using Spearman's rank-order.

To enable reproducibility of our work, we will make available all the anonymised pre-surgery and surgically-spared brain networks of 51 patients, brain networks of 29 controls, codes for node abnormality computations, and all the trained machine learning models on the data presented in our manuscript (link to be generated upon acceptance of the manuscript). 


\section{Results}

The results are organised in three parts. First, we assessed if patients with greater number of abnormal nodes are predisposed to have suboptimal seizure outcome after surgery. Second, we investigated the effect of surgery in reducing the node abnormality load between the seizure-free and non-seizure groups. Third, we determined the generalisability of node abnormality measure, if it is to be incorporated in a clinical setting alongside other clinical attributes, to estimate the chances of seizure recurrence for new patients. The overall pipeline is shown in Figure 2 and explained in Methods. The full clinical data for each patient is provided in Table S1.

\section{Abnormality load corresponds with surgical outcome and post-surgery seizure relapse}

We investigated the abnormality load in surgically-spared and pre-surgical networks. Figure 3(a-d) illustrates abnormal nodes in the surgically-spared networks for four patients. The patients in panels (a) and (b) were seizure free (ILAE 1) and having auras (ILAE 2) respectively at one-year after surgery and did not relapse subsequently; they had a relatively low node abnormality load. The patient in panel (c) initially had auras only (ILAE2) at one-year post-surgery but later relapsed; this patient showed a higher abnormal node count in the surgically-spared network. The patient in panel (d), with the highest number of abnormal nodes, had the worst surgical outcome of ILAE 5 at one year, which persisted upon follow-up. In these four cases a greater abnormality load was associated with seizure relapse, and worse outcomes. 
Figure 3(e) shows the node abnormality load in surgically-spared network for the entire patient cohort.

Patients who were not seizure free (ILAE 3-6) at one-year post surgery, had significantly more number of abnormal nodes than patients who were seizure free $(p=0.002, d=0.8$ between ILAE 1 and ILAE 3-6 and $p=0.009, d=0.6$ between ILAE 2 and ILAE 3-6). Here, we chose to analyse ILAE 2 as a separate group because clinical data (Table 1, S1) suggests that these patients, albeit free from disabling seizures at year-one, have a greater propensity to relapse in later years (Fairclough et al., 2018). Studying only the subset of patients who were seizure-free (i.e. ILAE 1, 2) at 1 year (Figure 3f-g), patients who relapsed had more abnormal nodes than the patients who did not relapse $(p=0.04, d=$ 0.75). Therefore, node abnormality can discriminate the patients in which seizures continued or recurred after surgery from the patients who were free from disabling seizures.

\section{[Figure 3]}

Node abnormality in Figure 3, computed from the surgically-spared network, was defined as the nodes with at least $10 \%$ of abnormal $(z>2.8)$ connections. At this choice of thresholds, the discrimination (AUC) between the seizure free and non-seizure free group was the highest. Comparable results are found for other threshold values (Supplementary Figure S1), and with an alternative network parcellation (Supplementary Figure S4). Thus, the discriminatory ability of node abnormality measure is consistent across the choice of threshold or the choice of parcellation scheme.

We found similar results in the pre-surgery networks. ILAE 3-6 patients had significantly more abnormal nodes than ILAE 1 patients $(p=0.02)$. However, the size of this effect was less pronounced than in the surgically-spared networks, with relatively poorer discriminatory ability (Supplementary Figures S1, and S2). Therefore, our findings suggest that the surgically-spared network, which is the surgically 
informed subnetwork of the pre-surgery network, is more discriminatory in identifying seizure free from non-seizure free patients.

\section{Surgery-related effect on reducing abnormality load}

How much effect does surgery have on reducing the abnormality load? We investigated the differences between the surgically-spared, and pre-surgery networks in terms of their abnormality load, and whether the projected change in abnormality load due to surgery was greater and more widespread in one outcome group compared to another. The proportion of abnormal nodes in different brain areas for ILAE 1 (seizure free) and ILAE 3-6 (not seizure free) groups are shown in Figure 4.

\section{[Figure 4]}

In terms of the spatial extent of surgery, the expected reduction in the proportion of abnormal nodes was more widespread in the seizure free group than in the non-seizure free group. The ILAE 1 group had a drop in the proportion of abnormal nodes in the surgically-spared network, compared to the presurgical network, in seven areas: five ipsilateral and two contralateral (Figure 4a-b). In ILAE 3-6 group, however, the drop in the proportion of abnormal nodes was limited to three ipsilateral areas: temporal, occipital, and frontal (Figure 4c-d). Similar surgery related effect was found for node abnormality computed at different threshold values (Supplementary Figure S3).

In terms of reduction in the amount of abnormality load, ILAE 1 patients had larger proportional reductions than ILAE3-6 patients $(p=0.01)$, however, their absolute reduction did not differ significantly ( $p=0.28$ ) (Supplementary Figure S5). Thus, we suggest that the temporal lobe epilepsy 
surgery causes a greater and widespread reduction in abnormality load in the seizure free group than in the non-seizure free group.

\section{Personalised prediction of unsuccessful surgeries}

We assessed the generalisability of the abnormality measure when used alongside other clinical attributes to predict patient-specific chances of poorer outcomes. Implementing nested-cross validation, we built machine learning models which classified new unseen (test) patients as either belonging to ILAE 1 or ILAE 3-6 group at 12 months. The model also scored each patient with a probability of belonging to either of the classes. Notably, the models were blind to three aspects of the data (a) all ILAE 2 patients, (b) ILAE classification 3, 4, 5 (the model simply sees these as 'poor outcome'), and (c) outcomes at later years.

\section{[Figure 5]}

We incorporated up to 15 features in the model: 13 clinical attributes, the pre-surgical abnormality load, and the surgically-spared abnormality load. These features describe the presurgical attributes of patients and we evaluated them based on their combined ability in accurately predicting surgical outcomes at one-year. However, some features may be less informative than others in predicting surgical outcomes; including less informative features causes a drop in the prediction performance. Therefore, by implementing stepwise removal of less informative features, we obtained combinations of preoperative features that identified patients with poor seizure outcome at one-year after surgery in $100 \%$ cases (i.e., specificity). The area under the ROC curve (AUC) at every step of feature elimination is plotted in Figure 5(a) and magnified at one example point (marked with a star) in Figure 5(b) with 
the corresponding confusion matrix shown in the inset. Average prediction performance across all stepwise feature removals was robust at; $A \cup C=0.84 \pm 0.07$, accuracy $=0.79 \pm 0.05$, specificity $=0.89$ \pm 0.09 , sensitivity $=0.77 \pm 0.06$. Supplementary Table 2 tabulates these prediction metrics in classifying seizure free and non-seizure free outcomes at every step. The lower panel in Figure 5(a) maps feature importance after each iteration of feature removal. The node abnormality in the surgically-spared network stood out as the most informative feature; it was more than 1.5 standard deviations away from the next most important features: age at surgery, and number of AEDs taken before surgery. Thus, including the abnormality measures to characterise pre-surgical attributes of intractable TLE patients led to a high and robust classification performance in predicting surgical outcomes at one-year after surgery.

We next analysed the scores/probabilities assigned by the model to each patient to have a non-seizure free surgical outcome. Larger probabilities indicated a greater predicted likelihood of postoperative seizure at year 1 (i.e., the ILAE3+ group). Since the model was trained only on binary ILAE1 and ILAE36 outcomes it was blind to the spectrum of ILAE class data. We found that despite being blinded to such information, the predicted likelihood of seizure relapse was positively correlated with ILAE surgical outcome scale at year-one (Figure $5 c$ ). This positive association is consistent, even for the model trained using only the surgically-spared node abnormality feature (Figure 5a). Spearman's rho values are plotted at each step-wise removal of features in Figure 5(a) and magnified for an example point in Figure 5(c). To confirm this result, we applied robust regression to obtain the regression slope and tested the significance of the steepness of the regression slope using permutation test (1000 permutations, $p=0.004$ in Supplementary Figure S6). Therefore, our result shows that the pre- 
surgical clinical profile of patients, when assessed along with the abnormality measures, can inform about the grade of seizure outcomes which a patient would expect after surgery.

\section{[Figure 6]}

How informative are the pre-surgical features in predicting seizure recurrences in the long-term? We analysed this by checking the association between the predicted likelihood of seizure relapse and the actual relapse data for patients who were seizure free (ILAE 1,2) at year one. Patients who were not seizure free at year one (ILAE 3-6) were not included in the relapse category. We found no association with seizure relapse when the pre-surgical features of patients were characterized using a combination of clinical and network abnormality measures (Supplementary Figure S7). However, significant association with relapse was present at year 3,4 , and 5 when the pre-surgical features of patients were characterized using only the abnormality load in surgically-spared network (Figure 6). Hence, we suggest that in determining the long-term seizure recurrences, pre-surgical clinical attributes are less informative than the measure of abnormality load expected to be present in a patient after surgery.

In summary, we achieved excellent performance in predicting seizure outcomes at one year when intractable TLE patients were assessed based on abnormality measures and clinical attributes. This combined pre-surgical profiling of patient attributes was also informative about the grades of seizure outcomes (ILAE class) at year one. However, beyond the first year after surgery, node abnormality in the surgically-spared networks were more informative about seizure relapses. 


\section{Discussion}

We investigated the association of surgical outcomes and relapse with the abnormality load computed in whole-brain pre-surgical network, and surgically spared subnetwork. Patients were more likely to have a poorer seizure outcome at one-year post surgery or a seizure relapse in five years, if more abnormal nodes were present in the surgically-spared network. Investigating the spatial effect of surgery on abnormality load, we found that the seizure free group of patients would have had a more widespread reduction of abnormal nodes. We found that the abnormality load in pre-surgery and surgically-spared networks, combined with clinical attributes of patients, generalised to predict 12month postoperative seizure freedom with $100 \%$ specificity and 0.91 AUC. With this combined characterization of patient attributes, we predicted the likelihood of seizure relapse patient-specifically which were correlated with the ILAE class, hence, informative of the seizure outcome expected at 12 months after surgery. Finally, we showed that node abnormality located in the surgically-spared networks were particularly informative in identifying patients who were initially seizure free but would relapse after the first year of surgery and up to 5 years.

Altered white-matter tract integrity, extending beyond the ipsilateral temporal lobe to the extratemporal and contralateral regions, has been extensively studied in TLE (Concha et al., 2012; Otte et al., 2012). Diffusion abnormalities with predictive value of postoperative outcomes are seen at the individual tract (Keller et al., 2017), and also at the whole-brain network level (Bonilha et al., 2013). It is indeed possible that areas which are anatomically normal, compared to controls, are epileptogenic via some other mechanism. Making an assumption that epileptogenic areas are most likely abnormal, our results show the presence of abnormal areas outside the temporal lobe which are spared by 
surgery. We suggest that net abnormality load, being a prognostic marker of seizure outcome, may be linked with epileptogenicity and have the potential to support epileptogenic networks.

A recent study on a different dataset with different imaging protocols, investigated network abnormality as a personalised predictor of surgical outcomes (Bonilha et al., 2015). In that study, presurgery networks were constructed based on the number of streamlines connecting different regions. Similar to our study, connections between ROls were normalised (z-transformed) against a control distribution. The similarity between our results suggest that: a) normalised patient networks using a local control distribution may enable reproducibility, comparison, and possibly grouping of patients between sites, and b) non-invasive personalised network biomarkers for predicting the likelihood of specific post-surgery outcomes in TLE are possible. We further showed the benefit of incorporating the information about the location of surgery to predict the surgical outcome.

The current standard for individualised prediction of surgical outcome primarily relies on clinical variables. However, there is considerable controversy in the literature regarding the presurgical clinical factors that may help predict surgical outcome. The review by (Bonilha and Keller, 2015) discussed discordant findings between different studies; features found predictive of seizure outcome in some studies are not predictive in others. Combining variables as nomograms, gave only modest concordance-statistics (c-statistics) of $\sim 0.5$ on validation models (Jehi et al., 2015). A more recent study estimated the probability of seizure freedom using combinations of up to 27 clinical variables on a mixed cohort of TLE and ETLE patients (Bell et al., 2017). Our findings indicated that combining clinical variables with brain connectome derived features such as: abnormality load in pre-surgery and surgically-spared networks, can improve prediction of surgical outcomes in the short-term. Particularly 
for long-term predictions, the abnormalities in the surgically-spared networks, which are expected to remain after surgery, may be a more reliable measure because they associate with relapses. Hence, we propose to combine our node abnormality measure with clinical variables in a large mixed-cohort patient (Bell et al., 2017) to improve estimation of the probability of seizure freedom/relapse after surgery.

In combining multivariate data, machine learning techniques delineate, rank, and fit input features of the training set to draw a decision boundary in a high dimensional space that maximises prediction (Bonilha et al., 2015; Munsell et al., 2015; Gleichgerrcht et al., 2018; Taylor et al., 2018; Morgan et al., 2019). While a binary classification of seizure free and non-seizure free outcomes at 12 months is important, predicting long-term trajectories of seizure freedom is also crucial to inform clinical management decisions. In our study, the classifier not only predicted the surgical outcome at one-year but also predicted the likelihood of seizure relapse. This additional information may be clinically useful for advising patients about their chances of poor outcome post-surgery beyond the first 12 months and represents a key novelty of our work.

The outcome of epilepsy surgery will not just depend on the brain network before the surgery but also on the location and extent of surgery (Ji et al., 2015; Taylor et al., 2018). In this study, we retrospectively included the information of surgery by drawing a resection mask and inferring an expected surgicallyspared network. A limitation of our work is that we are only inferring the expected postoperative network, rather than deriving it from postoperative dMRI data (Winston et al., 2014). However, an analysis using actual postoperative dMRI data would only have very limited value in terms of improving the pre-operative decision making, since the outcome could only be seen after the surgery has been 
performed. In contrast, our approach can be used before the actual surgery to evaluate likelihood of success. A prospective application would involve drawing a resection mask for an intended surgery on sMRI of a patient acquired before surgery (Taylor et al., 2018). Surgical data (either retrospectively delineated or prospectively planned) enables the study of expected changes after surgery (Taylor et al., 2018). We showed that this information improves the prediction performance more so than just the pre-surgery networks which are naïve to surgical information. We envisage a software tool where multiple standard operations could be selected (e.g. selective amygdalohippocampectomy, or anterior temporal lobe resection) and their impact on the abnormality load compared. Tailored resections could also be tested to see what the effect of a larger resection might be. Such a tool could then be used to prospectively guide decision making regarding personalised resection strategies.

With regard to the extent of surgical resection, (Schramm, 2008) showed that the amount of tissue resected does not necessarily relate to improved surgical outcome. What is included in the resection, however, may have a significant influence on outcome (Siegel et al., 1990; Bonilha et al., 2012). The question arises: will a tailored resection, designed to reduce the number of abnormal nodes, lead to a better outcome? While more investigations are needed to confirm this hypothesis, we found that the seizure free patient group had a more widespread reduction of abnormality load due to surgery. Simulated computer models may facilitate a more detailed analysis to investigate alternative surgical strategies in a personalised manner (Sinha et al., 2017).

Our findings must be interpreted in the context of some caveats. Node abnormality may be representative of a) network reorganisation in response to seizures, b) neurodegenerative process due to seizures, c) structures facilitating seizures, or combinations of (a)-(c). In our study we could not 
disentangle these aspects with respect to node abnormality. We did not detect any significant correlation between clinical variables and node abnormalities. Though our sample size is reasonably large (Bonilha et al., 2015; Munsell et al., 2015; Keller et al., 2017; Gleichgerrcht et al., 2018), it is not of the size of typical epidemiological studies. Neural architecture depends on several subject-specific factors including language dominance, handedness, and other physiological variables. These relationships may further influence the node abnormality measure. Thus, our results should motivate a larger study to test its generalisability, ideally across multiple sites. Finally, we highlight, based on the pre-surgical, surgically-spared networks, and clinical variables, the chances of at least one relapse in five-years. However, the trajectory of seizure remission and relapse is more complicated. Patients may have repeated remissions and relapses due to drug-effects, environmental factors, or other causes.

In summary, we have shown evidence of node abnormality being an important non-invasive marker of surgical outcome and its severity at year one post-surgery. Node abnormality is also related with likelihood of seizure relapse in long-term. We demonstrate improvement in prediction performance when including surgery information with the pre-surgery network and clinical data. We believe this to be an important step towards complementing clinical decision making on patient and surgery selection for intractable TLE as well as for patient counselling regarding the risks of seizure severity expected after surgery. 


\section{Bibliography}

Bell GS, de Tisi J, Gonzalez-Fraile JC, Peacock JL, McEvoy AW, Harkness WFJ, et al. Factors affecting seizure outcome after epilepsy surgery: an observational series. J Neurol Neurosurg Psychiatry 2017; 88: 933-940.

Bernasconi N, Duchesne S, Janke A, Lerch J, Collins DL, Bernasconi A. Whole-brain voxel-based statistical analysis of gray matter and white matter in temporal lobe epilepsy. Neuroimage 2004

Bernhardt BC, Bonilha L, Gross DW. Network analysis for a network disorder: The emerging role of graph theory in the study of epilepsy. Epilepsy Behav 2015; 50: 162-170.

Besson P, Bandt SK, Proix T, Lagarde S, Jirsa VK, Ranjeva J-P, et al. Anatomic consistencies across epilepsies: a stereotactic-EEG informed high-resolution structural connectivity study. Brain 2017; 140: 2639-2652.

Bonilha L, Helpern JA, Sainju R, Nesland T, Edwards JC, Glazier SS, et al. Presurgical connectome and postsurgical seizure control in temporal lobe epilepsy. Neurology 2013; 81: 1704-1710.

Bonilha L, Jensen JH, Baker N, Breedlove J, Nesland T, Lin JJ, et al. The brain connectome as a personalized biomarker of seizure outcomes after temporal lobectomy. Neurology 2015; 84: 18461853.

Bonilha L, Keller SS. Quantitative MRI in refractory temporal lobe epilepsy: relationship with surgical outcomes. Quant Imaging Med Surg 2015; 5: 204-24.

Bonilha L, Martz GU, Glazier SS, Edwards JC. Subtypes of medial temporal lobe epilepsy: Influence on temporal lobectomy outcomes? Epilepsia 2012; 53: 1-6.

Cohen-Gadol AA, Wilhelmi BG, Collignon F, White JB, Britton JW, Cambier DM, et al. Long-term outcome of epilepsy surgery among 399 patients with nonlesional seizure foci including mesial temporal lobe sclerosis. J Neurosurg 2008; 104: 513-524.

Concha L, Kim H, Bernasconi A, Bernhardt BC, Bernasconi N. Spatial patterns of water diffusion along white matter tracts in temporal lobe epilepsy. Neurology 2012; 79: 455-462.

Deleo F, Thom M, Concha L, Bernasconi A, Bernhardt B, Bernasconi N. Histological and MRI markers of white matter damage in focal epilepsy. Epilepsy Res 2017 doi: 10.1016/j.eplepsyres.2017.11.010

Fairclough S, O'Keeffe AG, De Tisi J, Duncan JS. Auras and the risk of seizures with impaired consciousness following epilepsy surgery: Implications for driving. J Neurol Neurosurg Psychiatry 2018; 89: 599-602.

Fagerholm ED, Hellyer PJ, Scott G, Leech R, Sharp DJ. Disconnection of network hubs and cognitive impairment after traumatic brain injury. Brain 2015; 138: 1696-1709.

Gleichgerrcht E, Munsell B, Bhatia S, Vandergrift WA, Rorden C, McDonald C, et al. Deep learning 
applied to whole-brain connectome to determine seizure control after epilepsy surgery. Epilepsia 2018; 59: 1643-1654.

Goodfellow M, Rummel C, Abela E, Richardson MP, Schindler K, Terry JR. Estimation of brain network ictogenicity predicts outcome from epilepsy surgery. Sci Rep 2016; 6: 29215.

Guyon I, Elisseeff A. An Introduction to Variable and Feature Selection. J Mach Learn Res 2003; 3: 11571182.

Guyon I, Weston J, Barnhill S, Vapnik V. Gene selection for cancer classification using support vector machines. Mach Learn 2002

Haneef Z, Stern J, Dewar S, Engel J. Referral pattern for epilepsy surgery after evidence-based recommendations: A retrospective study. Neurology 2010; 75: 699-704.

Janszky J, Janszky I, Schulz R, Hoppe M, Behne F, Pannek HW, et al. Temporal lobe epilepsy with hippocampal sclerosis: Predictors for long-term surgical outcome. Brain 2005

Jehi L, Yardi R, Chagin K, Tassi L, Russo G Lo, Worrell G, et al. Development and validation of nomograms to provide individualised predictions of seizure outcomes after epilepsy surgery: a retrospective analysis. Lancet Neurol 2015; 14: 283-290.

Ji G-J, Zhang Z, Xu Q, Wei W, Wang J, Wang Z, et al. Connectome Reorganization Associated With Surgical Outcome in Temporal Lobe Epilepsy. Medicine (Baltimore) 2015; 94: e1737.

Keller SS, Glenn GR, Weber B, Kreilkamp BAK, Jensen JH, Helpern JA, et al. Preoperative automated fibre quantification predicts postoperative seizure outcome in temporal lobe epilepsy. Brain 2017; 140 : 68-82.

Keller SS, Roberts N. Voxel-based morphometry of temporal lobe epilepsy: An introduction and review of the literature. Epilepsia 2008; 49: 741-757.

Langfitt JT, Wiebe S. Early surgical treatment for epilepsy. Curr Opin Neurol 2008

Li W, An D, Tong X, Liu W, Xiao F, Ren J, et al. Different patterns of white matter changes after successful surgery of mesial temporal lobe epilepsy. Neurolmage Clin 2019; 21: 101631.

Liu M, Bernhardt BC, Hong S-J, Caldairou B, Bernasconi A, Bernasconi N. The superficial white matter in temporal lobe epilepsy: a key link between structural and functional network disruptions. Brain 2016; 139: 2431-2440.

McDonald CR, Hagler DJ, Ahmadi ME, Tecoma E, Iragui V, Gharapetian L, et al. Regional neocortical thinning in mesial temporal lobe epilepsy. Epilepsia 2008; 49: 794-803.

Morgan VL, Englot DJ, Rogers BP, Landman BA, Cakir A, Abou-Khalil BW, et al. Magnetic resonance imaging connectivity for the prediction of seizure outcome in temporal lobe epilepsy. Epilepsia 2017; 58: 1251-1260.

Morgan VL, Rogers BP, Anderson AW, Landman BA, Englot DJ. Divergent network properties that 
predict early surgical failure versus late recurrence in temporal lobe epilepsy. J Neurosurg 2019; $1: 1-$ 10.

Munsell BC, Wee C-Y, Keller SS, Weber B, Elger C, da Silva LAT, et al. Evaluation of machine learning algorithms for treatment outcome prediction in patients with epilepsy based on structural connectome data. Neuroimage 2015; 118 IS-: 219-230.

Otte WM, van Eijsden P, Sander JW, Duncan JS, Dijkhuizen RM, Braun KPJ. A meta-analysis of white matter changes in temporal lobe epilepsy as studied with diffusion tensor imaging. Epilepsia 2012; 53: 659-667.

Platt JC. Probabilistic outputs for support vector machines and comparisons to regularized likelihood methods. Adv large margin Classif 1999

Proix T, Bartolomei F, Guye M, K JV. Individual brain structure and modelling predict seizure propagation [Internet]. Brain 2017Available from: https://doi.org/10.1093/brain/awx004

de Reus MA, van den Heuvel MP. Estimating false positives and negatives in brain networks. Neuroimage 2013; 70: 402-409.

Richardson MP. Large scale brain models of epilepsy: dynamics meets connectomics. J Neurol Neurosurg Psychiatry 2012; 83: 1238-1248.

Schramm J. Temporal lobe epilepsy surgery and the quest for optimal extent of resection: A review. Epilepsia 2008.

Siegel AM, Wieser HG, Wichmann W, Yasargil GM. Relationships between MR-imaged total amount of tissue removed, resection scores of specific mediobasal limbic subcompartments and clinical outcome following selective amygdalohippocampectomy. Epilepsy Res 1990

Sinha N, Dauwels J, Kaiser M, Cash SS, Westover MB, Wang Y, et al. Predicting neurosurgical outcomes in focal epilepsy patients using computational modelling. Brain 2017; 140

Smialowski P, Frishman D, Kramer S. Pitfalls of supervised feature selection. Bioinformatics 2009

Spencer S, Huh L. Outcomes of epilepsy surgery in adults and children. Lancet Neurol 2008; 7: 525537.

Spencer SS. Neural Networks in Human Epilepsy: Evidence of and Implications for Treatment. Epilepsia 2002; 43: 219-227.

Taylor PN, Sinha N, Wang Y, Vos SB, de Tisi J, Miserocchi A, et al. The impact of epilepsy surgery on the structural connectome and its relation to outcome. Neurolmage Clin 2018; 18

Téllez-Zenteno JF, Wiebe S. Long-term seizure and psychosocial outcomes of epilepsy surgery. Curr Treat Options Neurol 2008; 10: 253-259.

de Tisi J, Bell GS, Peacock JL, McEvoy AW, Harkness WFJ, Sander JW, et al. The long-term outcome of adult epilepsy surgery, patterns of seizure remission, and relapse: a cohort study. Lancet (London, 
England) 2011; 378: 1388-1395.

Tsamardinos I, Greasidou E, Borboudakis G. Bootstrapping the out-of-sample predictions for efficient and accurate cross-validation. Mach Learn 2018

Tuch DS. Q-ball imaging. Magn Reson Med 2004; 52: 1358-1372.

Vakharia VN, Duncan JS, Witt JA, Elger CE, Staba R, Engel J. Getting the best outcomes from epilepsy surgery. Ann Neurol 2018Available from: https://onlinelibrary.wiley.com/doi/full/10.1002/ana.25205

Wiebe S, Blume WT, Girvin JP, Eliasziw M. A Randomized, Controlled Trial of Surgery for Temporal-Lobe Epilepsy. N Engl J Med 2001; 345: 311-318.

Wieser HG, Blume WT, Fish D, Goldensohn E, Hufnagel A, King D, et al. Proposal for a New Classification of Outcome with Respect to Epileptic Seizures Following Epilepsy Surgery. Epilepsia 2001; 42: 282286.

Winston, Gavin P., Jason Stretton, Meneka K. Sidhu, Mark R. Symms, and John S. Duncan. "Progressive white matter changes following anterior temporal lobe resection for epilepsy." Neurolmage: Clinical 4 (2014): 190-200.

\section{Acknowledgements}

NS was supported by Research Excellence Academy, Newcastle University, UK. PNT was supported by the Wellcome Trust (105617/Z/14/ Z and 210109/Z/18/Z). YW was supported by the Wellcome Trust (208940/Z/17/Z). We thank Gabrielle Schroeder, Pawel Widera, Sriharsha Ramaraju, other members of ICOS, and CNNP lab (www.cnnp-lab.com) for discussions. SBV was funded by the UCLH NIHR BRC. Scan acquisition and GPW were supported by the MRC (G0802012, MR/M00841X/1). We are grateful to the Epilepsy Society for supporting the Epilepsy Society MRI scanner. This work was supported by the National Institute for Health Research University College London Hospitals Biomedical Research Centre. 
Table 1: Demographic and clinical data of patients

\begin{tabular}{|c|c|c|c|c|}
\hline $\begin{array}{l}\text { Year } 1 \text { outcome } \\
\text { Variables }\end{array}$ & ILAE 1 & ILAE 2 & ILAE 3-6 & Significance \\
\hline Patients (n) & 34 & 8 & 9 & \\
\hline $\begin{array}{r}\text { Sex } \\
(\text { Male/Female) }\end{array}$ & $16 / 18$ & $2 / 6$ & $2 / 7$ & $\begin{array}{l}\chi_{1,2}^{2}=0.54, \mathrm{p}_{1,2}=0.46 \\
\chi_{1,3+}^{2}=0.93, \mathrm{p}_{1,3+}=0.33 \\
\chi^{2}{ }_{2,3+}=0.19, \mathrm{p}_{2,3+}=0.66\end{array}$ \\
\hline $\begin{array}{r}\text { Age at Onset } \\
\text { (mean } \pm \text { std) }\end{array}$ & $12.2 \pm 10.3$ & $14.2 \pm 11.4$ & $19 \pm 12$ & $\begin{array}{l}p_{1,2}=0.62 \\
p_{1,3+}=0.04 \\
p_{2,3+}=0.43\end{array}$ \\
\hline $\begin{array}{r}\text { Age at Surgery } \\
\text { (mean } \pm \text { std) }\end{array}$ & $38 \pm 11.9$ & $38.6 \pm 10.3$ & $46.5 \pm 10.2$ & $\begin{array}{l}p_{1,2}=0.96 \\
p_{1,3+}=0.08 \\
p_{2,3+}=0.13\end{array}$ \\
\hline $\begin{array}{r}\text { Epilepsy Duration } \\
\text { (mean } \pm \text { std) }\end{array}$ & $25.8 \pm 15.8$ & $24.3 \pm 17.3$ & $27.5 \pm 7.3$ & $\begin{array}{l}p_{1,2}=0.74 \\
p_{1,3+}=0.54 \\
p_{2,3+}=0.54\end{array}$ \\
\hline $\begin{array}{r}\text { Side } \\
\text { (Left/Right) }\end{array}$ & $22 / 12$ & $3 / 5$ & $5 / 4$ & $\begin{array}{l}\chi_{1,2}^{2}=1.02, \mathrm{p}_{1,2}=0.31 \\
\chi^{2}{ }_{1,3+}=0.01, \mathrm{p}_{1,3+}=0.91 \\
\chi^{2}{ }_{2,3+}=0.06, \mathrm{p}_{2,3+}=0.79\end{array}$ \\
\hline Hippocampal sclerosis & 24 (70.5\%) & $6(75 \%)$ & 5 (55.5\%) & $\begin{array}{l}\chi^{2}{ }_{1,2}=0.03, \mathrm{p}_{1,2}=0.85 \\
\chi^{2}{ }_{1,3+}=0.21, \mathrm{p}_{1,3+}=0.64 \\
\chi^{2}{ }_{2,3+}=0.11, \mathrm{p}_{2,3+}=0.74\end{array}$ \\
\hline $\begin{array}{r}\text { Number of AEDs before } \\
\text { surgery (mean } \pm \text { std) }\end{array}$ & $6.3 \pm 2.4$ & $7.6 \pm 2.9$ & $9.2 \pm 3.3$ & $\begin{array}{l}p_{1,2}=0.10 \\
p_{1,3+}=0.01 \\
p_{2,3+}=0.46\end{array}$ \\
\hline $\begin{array}{r}\text { Preoperative MRI } \\
\text { (Normal/Abnormal) }\end{array}$ & $5 / 29$ & $1 / 7$ & $2 / 7$ & $\begin{array}{l}\chi_{1,2}^{2}=0.16, \mathrm{p}_{1,2}=0.69 \\
\chi_{1,3+}^{2}=0.001, \mathrm{p}_{1,3+}=0.97 \\
\chi_{2,3+}^{2}=0.01, \mathrm{p}_{2,3+}=0.91\end{array}$ \\
\hline $\begin{array}{r}\text { History of status } \\
\text { epilepticus }\end{array}$ & 5 (15.7\%) & $0(0 \%)$ & $3(33.3 \%)$ & $\begin{array}{l}\chi_{1,2}^{2}=0.30, \mathrm{p}_{1,2}=0.58 \\
\chi_{1,3+}^{2}=0.63, \mathrm{p}_{1,3+}=0.43 \\
\chi_{2,3+}^{2}=1.35, \mathrm{p}_{2,3+}=0.25\end{array}$ \\
\hline $\begin{array}{r}\text { Secondary generalised } \\
\text { seizures }\end{array}$ & $28(82.3 \%)$ & $6(75 \%)$ & $6(66.7 \%)$ & $\begin{array}{l}\chi_{1,2}^{2}=0.0005, \mathrm{p}_{1,2}=0.98 \\
\chi_{1,3+}^{2}=0.32, \mathrm{p}_{1,3+}=0.57 \\
\chi_{2,3+}^{2}=0.02, \mathrm{p}_{2,3+}=0.88\end{array}$ \\
\hline Depression & 8 & 5 & 2 & $\chi^{2}{ }_{1,2}=2.96, \mathrm{p}_{1,2}=0.09$ \\
\hline
\end{tabular}




\begin{tabular}{|r|c|c|c|c|}
\hline & & & $\begin{array}{l}\chi_{1,3+}^{2}=0.13, \mathrm{p}_{1,3+}=0.72 \\
\chi_{2,3+}^{2}=1.42, \mathrm{p}_{2,3+}=0.23\end{array}$ \\
\hline Psychosis & 2 & 0 & 0 & $\begin{array}{l}\chi^{2}{ }_{1,2}=0.05, \mathrm{p}_{1,2}=0.83 \\
\chi_{1,3+}^{2}=0.02, \mathrm{p}_{1,3+}=0.88\end{array}$ \\
\hline $\begin{array}{r}\text { Other psychiatric } \\
\text { disorders }\end{array}$ & 8 & 3 & $\begin{array}{l}\chi^{2}{ }_{1,2}=0.13, \mathrm{p}_{1,2}=0.72 \\
\chi^{2}{ }_{1,3+}=0.68, \mathrm{p}_{1,3+}=0.41 \\
\chi_{2,3+}=0.04, \mathrm{p}_{2,3+}=0.84\end{array}$ \\
\hline $\begin{array}{r}\text { Relapsed by end of } \\
\text { Year } 2\end{array}$ & $0(0 \%)$ & $3(37.5 \%)$ & $9(100 \%)$ & \\
\hline $\begin{array}{r}\text { Relapsed by end of } \\
\text { Year 3 }\end{array}$ & $3(8.8 \%)$ & $5(62.5 \%)$ & $9(100 \%)$ & \\
\hline $\begin{array}{r}\text { Relapsed by end of } \\
\text { Year } 4\end{array}$ & $5(14.7 \%)$ & $6(75 \%)$ & $9(100 \%)$ & \\
\hline $\begin{array}{r}\text { Relapsed by end of } \\
\text { Year 5 }\end{array}$ & $7(20.6 \%)$ & $6(75 \%)$ & $9(100 \%)$ & \\
\hline
\end{tabular}



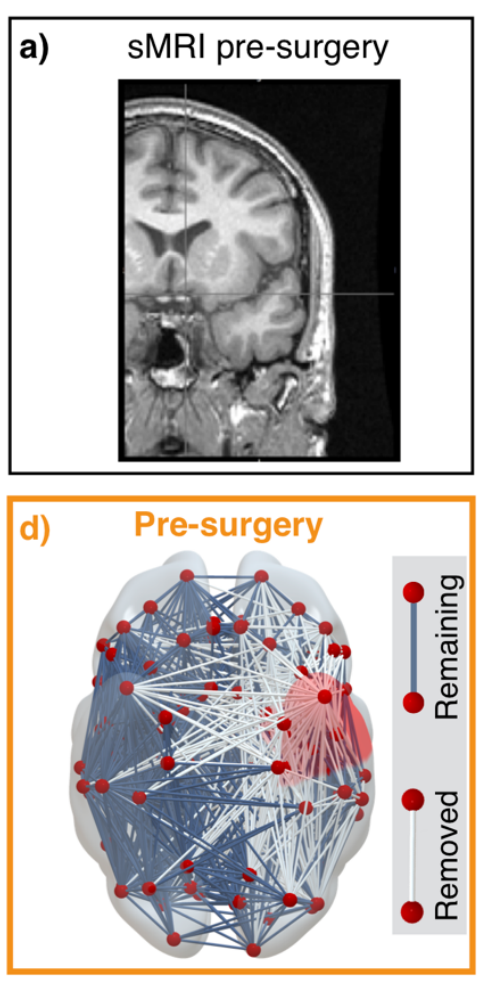
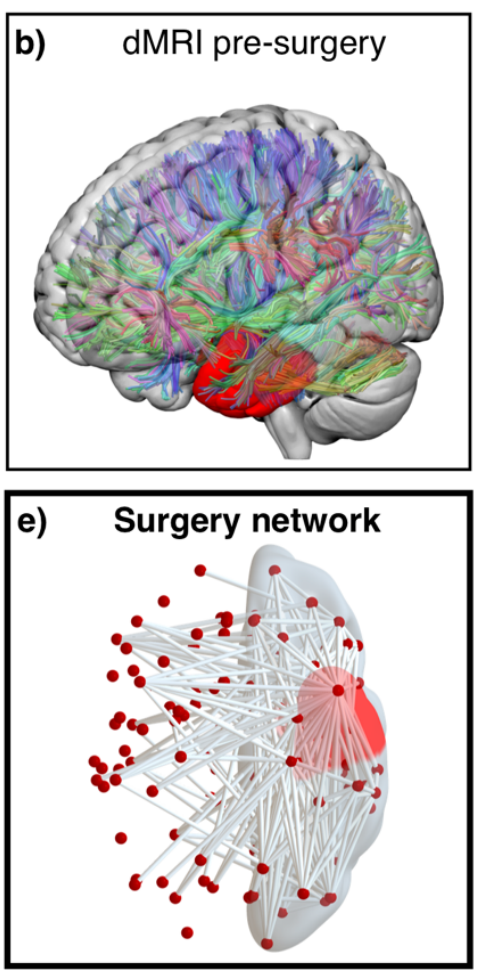
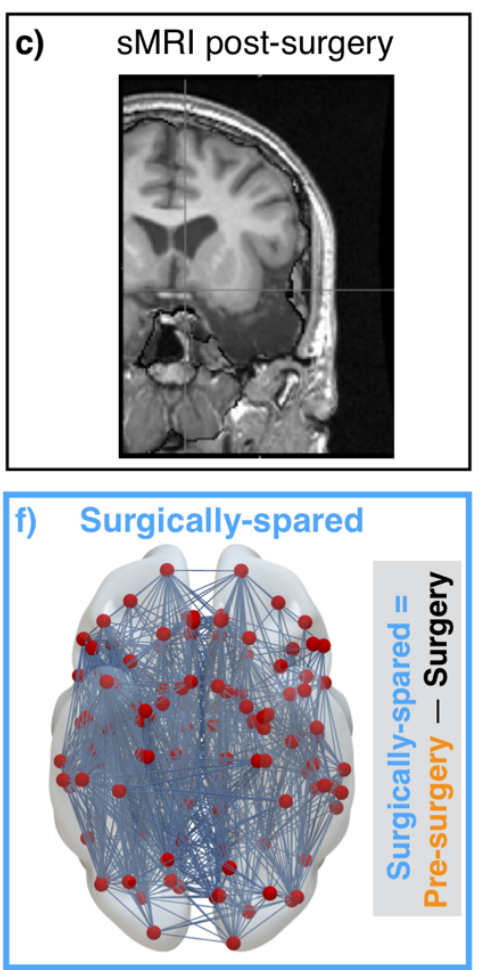

Figure 1: Estimating patient-specific surgery network. Preoperative T1w MRI of an example patient in

panel a) and postoperative T1w MRI in panel c) were used to delineate the tissue resected by surgery.

The resected tissue shown by the red resection mask in panel b) was used with the preoperative diffusion MRI to infer brain networks. Pre-surgery network inferred based on the number of streamlines connecting different ROIs in panel d) ignores the surgery information by not taking the resection mask into consideration. The patient-specific surgery network is illustrated in panel e) which shows the connections that were affected by the surgery. Panel f) shows the surgically-sparred subnetwork. 
bioRxiv preprint doi: https://doi.org/10.1101/747725; this version posted September 1, 2019. The copyright holder for this preprint (which was not certified by peer review) is the author/funder, who has granted bioRxiv a license to display the preprint in perpetuity. It is made available under aCC-BY-NC 4.0 International license.

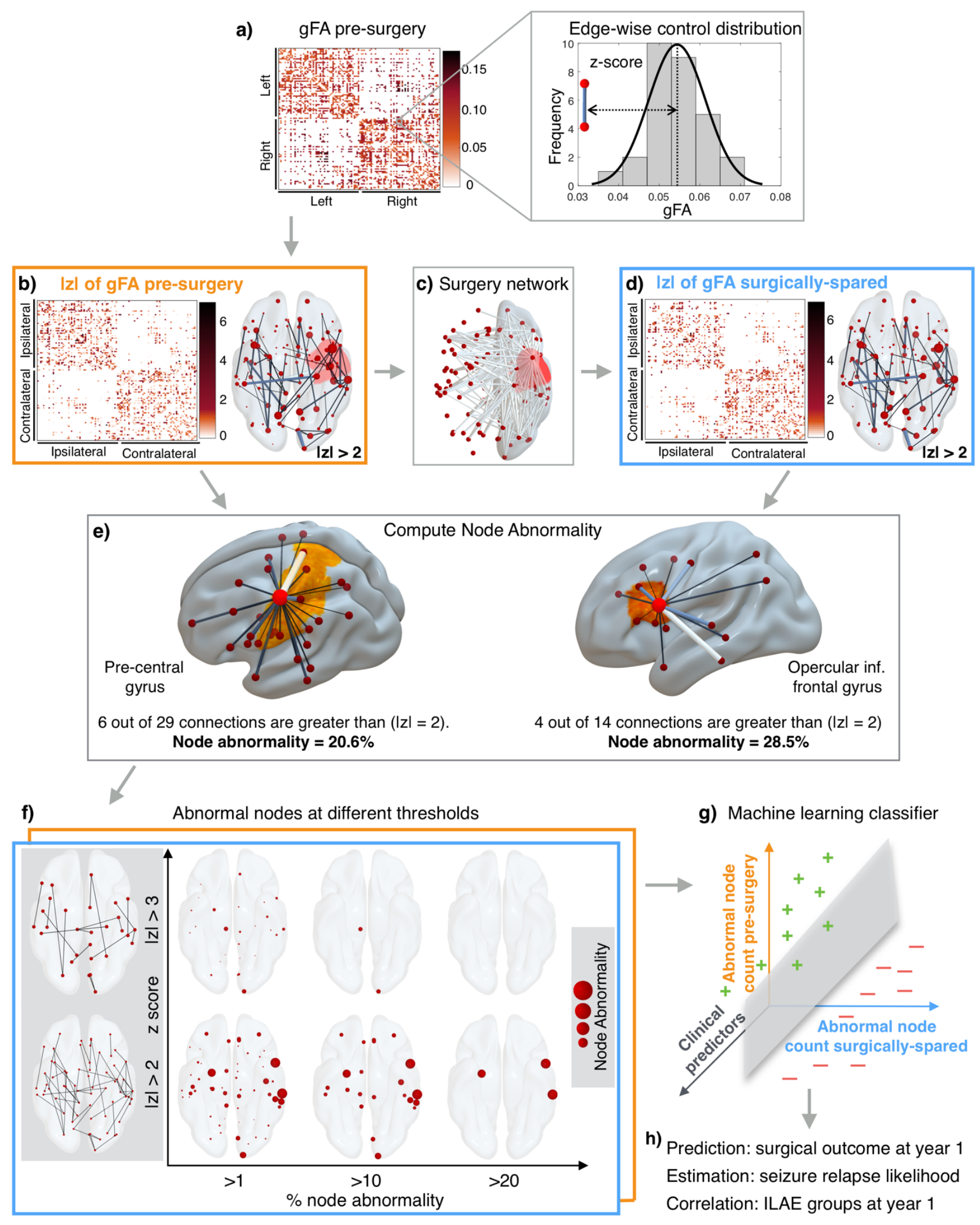


Figure 2: Overall pipeline. Pre-surgical gFA network architecture for each patient in panel a) is inferred and edges are standardised against a control distribution to obtain a z-score transformed network in panel b). The connections affected by the surgery shown in surgery network in panel c) are removed to obtain surgically-spared network in panel d). Panel e) shows the concept of node abnormality for two example nodes. By normalising the number of abnormal links to a node with its degree, the heterogeneity in the degree of network nodes is accounted for. A high degree node can be less abnormal compared to a low degree node depending on the number of abnormal connections. $\mathbf{f}$ ) Different thresholds required for the computation of node abnormality are shown. z-score at which a link is considered abnormal is on the $y$-axis and the cut-off at which a node is considered abnormal is shown on the x-axis. g-h) Abnormal node count in pre-surgery and surgically-spared networks are incorporated in a machine learning classifier along with the clinical predictors to predict surgical outcomes with its severity at year-one and estimate chances of seizure relapse in five years. 

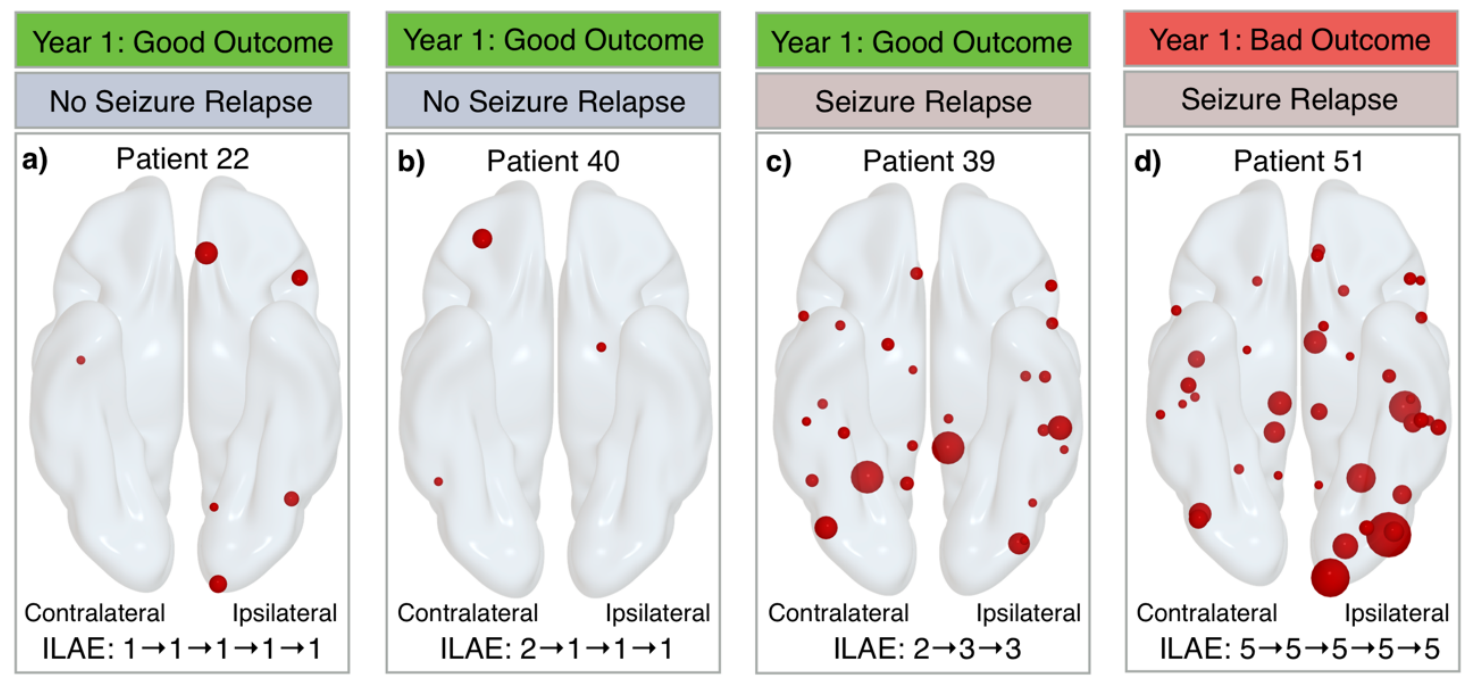

\section{0}

Node Abnormality
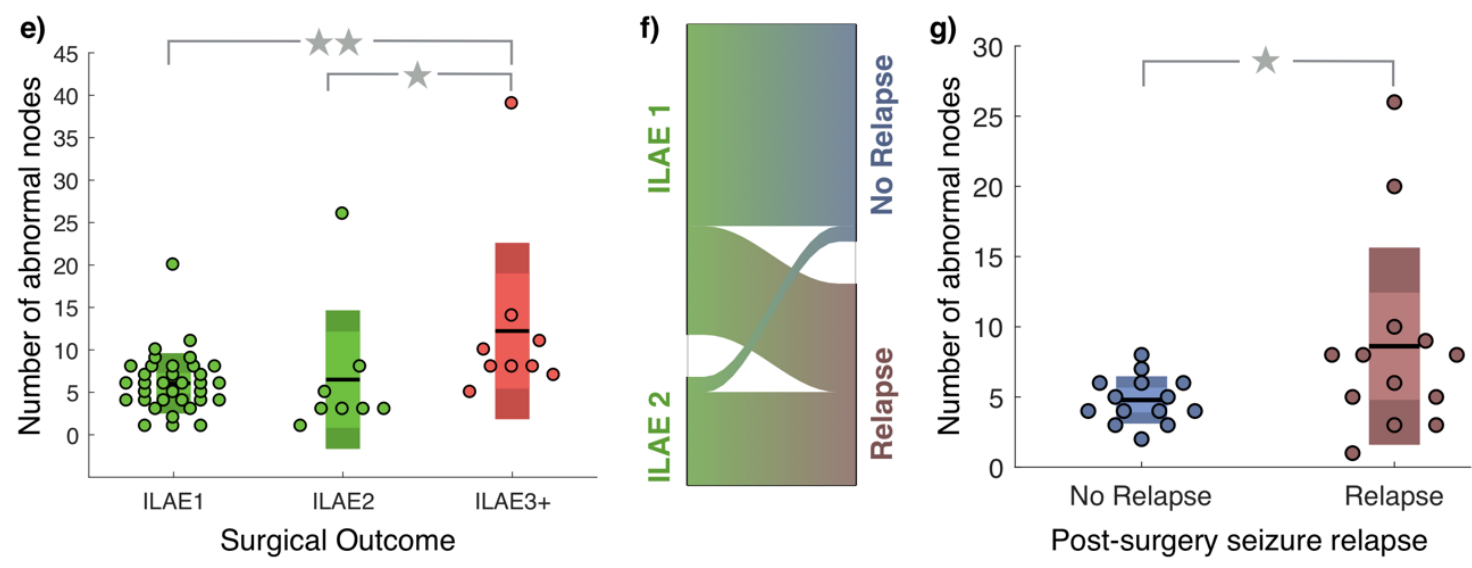

Figure 3: Association between the number of abnormal nodes in surgically-spared network with year-one surgical outcome and relapse. a-d) Four patients are shown with their year-one surgical outcome and relapse information. Panel a) and b) show fewer abnormal nodes in patients with ILAE 1 and ILAE 2 outcomes respectively with no relapse. Panel c) shows a patient with many abnormal nodes remaining yet having an ILAE 2 outcome at year-one but relapsing subsequently. Panel d) shows a large number of abnormal nodes remaining in a patient who was never seizure-free in five years. e) Significantly more abnormal nodes remained in ILAE 3+ patients compared to ILAE 1 and ILAE 2. f) Alluvial flow diagram showing proportion of relapsed patients with ILAE 1 or ILAE 2 at year-one. $g$ ) In 
bioRxiv preprint doi: https://doi.org/10.1101/747725; this version posted September 1, 2019. The copyright holder for this preprint (which was not certified by peer review) is the author/funder, who has granted bioRxiv a license to display the preprint in perpetuity. It is made available under aCC-BY-NC 4.0 International license.

ILAE 1-2 patients, those who relapsed had significantly more abnormal nodes in the surgically-spared network. 
ILAE 1

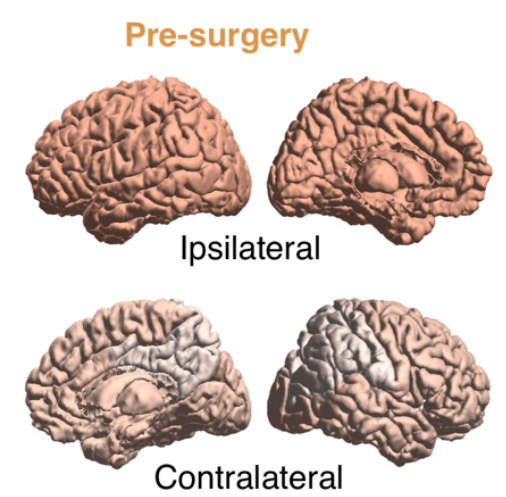

a)

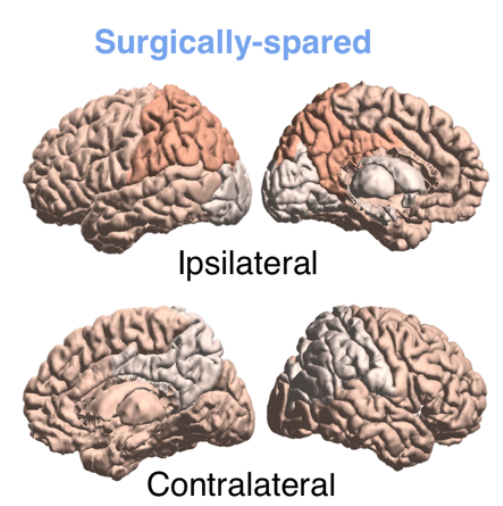

Contralateral

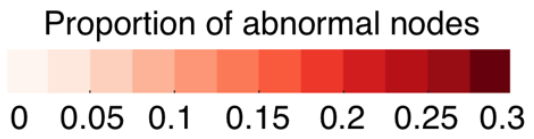

ILAE 3+

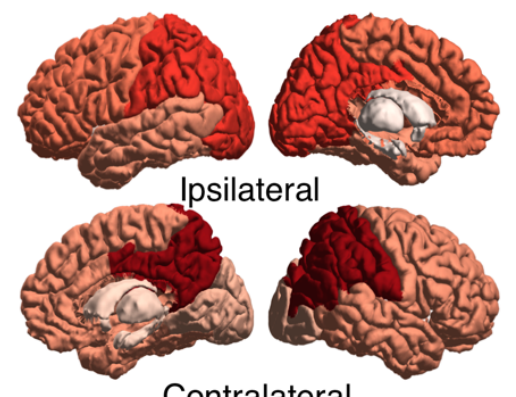

Contralateral

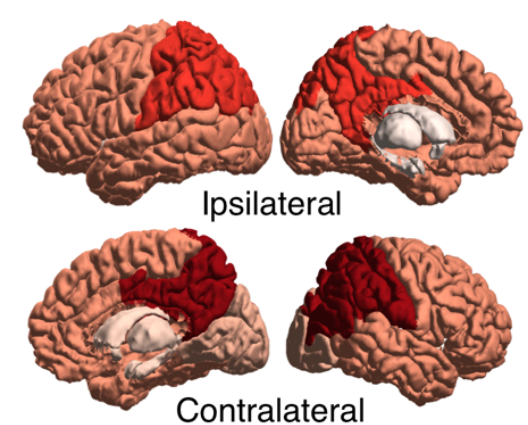

c)
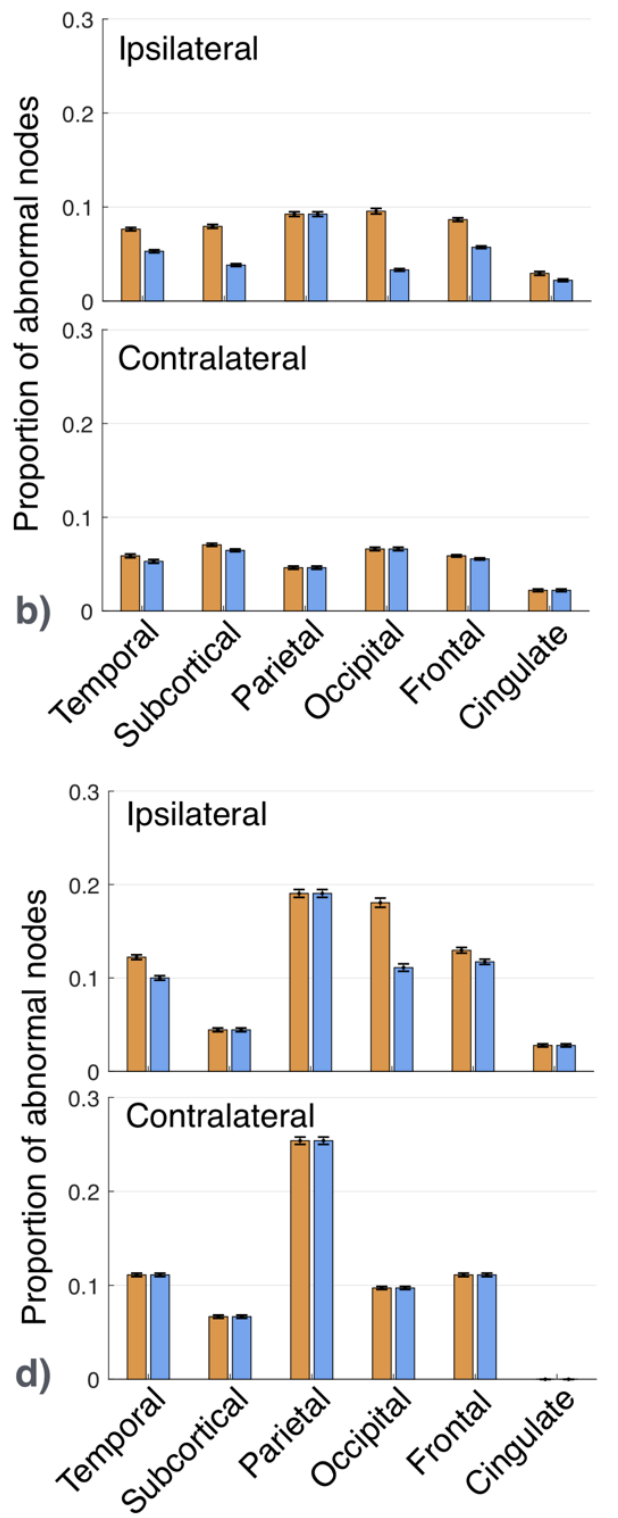

Figure 4: Effect of surgery in reducing node abnormality is more widespread in the seizure free group.

a) Proportion of abnormal nodes computed for pre-surgery and surgically-spared networks are colour coded for six ipsilateral and contralateral brain areas in the seizure free (ILAE 1) group. b) Bar plot shows the drop in surgically-spared network compared to pre-surgery network in five ipsilateral (temporal subcortical, occipital, frontal, and cingulate) and two contralateral (temporal and subcortical) areas in the seizure free (ILAE 1) group. Error bars represent the standard error of the 
bioRxiv preprint doi: https://doi.org/10.1101/747725; this version posted September 1, 2019. The copyright holder for this preprint (which was not certified by peer review) is the author/funder, who has granted bioRxiv a license to display the preprint in perpetuity. It is made available under aCC-BY-NC 4.0 International license.

proportion of abnormal nodes in each area. c) Different brain areas in pre-surgery and surgically-spared network are colour coded based on the proportion of abnormal nodes in the non-seizure-free group (ILAE 3+) d) Corresponding bar plot showing a drop in node abnormality in surgically-spared network in three ipsilateral areas: temporal, occipital, and frontal in the not-seizure-free group (ILAE 3+). 


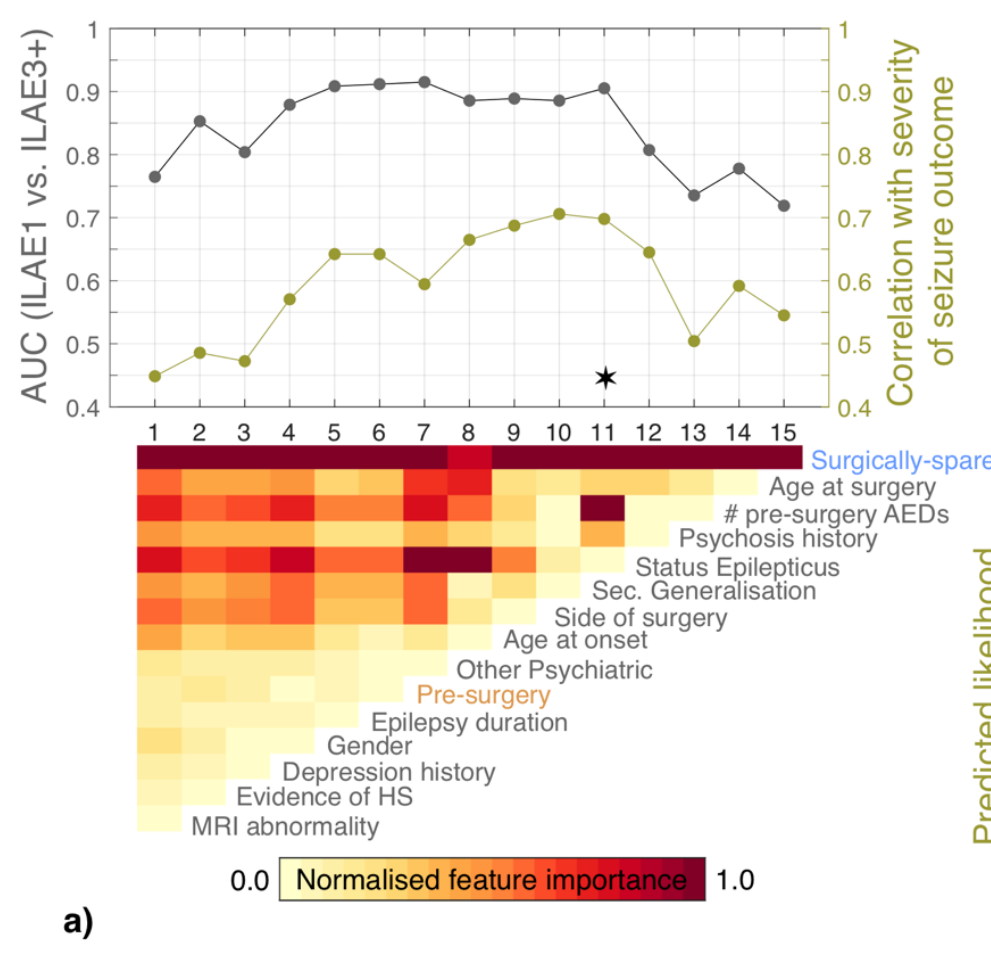

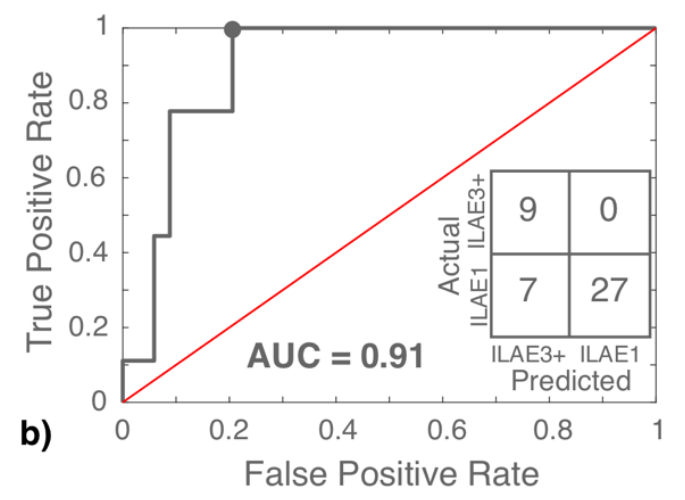

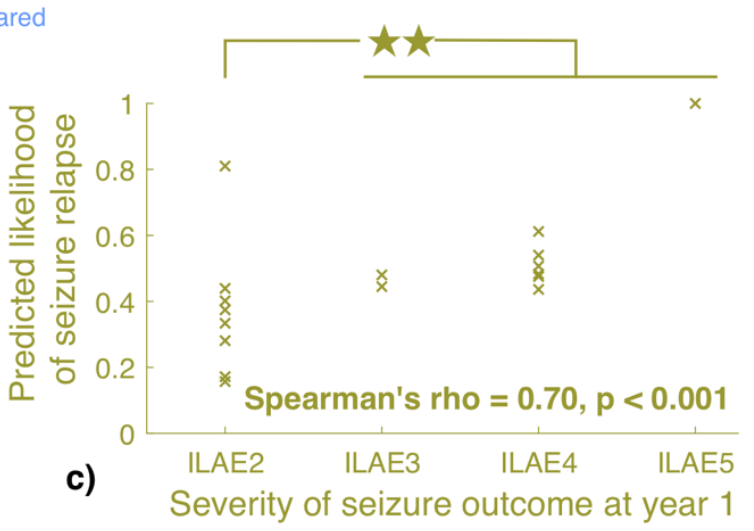

Figure 5: Prediction of seizure outcomes at year-one. a) AUC of SVMs that predicted seizure free (ILAE 1) and non-seizure free (ILAE3+) outcomes at one year after surgery are plotted in black. Blinded to the exact ILAE categories, the model predicted 12-month likelihood of seizure relapse for each patient. The Spearman's rank correlation between likelihood of seizure relapse and the severity of surgical outcomes (ILAE class) at year-one are plotted in green. The lower panel of a) shows the relative feature importance of each SVM on a normalised scale between 0 and 1 . The leftmost SVM, plotted at $x=1$, incorporated all 15 features (13 clinical, node abnormality in pre-surgery and surgically-spared networks) to predict seizure free (ILAE 1) and non-seizure free (ILAE3+) outcomes at one year after surgery. Amongst all features, the relative importance of surgically-spared node abnormality was the highest whereas the relative importance of MRI abnormality was the least. Therefore, in the next iteration at $x=2$, a new SVM was retrained using the 14 features, after removing the MRI abnormality 
feature. This stepwise removal of metrics was continued until only a single metric (surgically-spared node abnormality) remained. b) ROC curve is plotted at an example combination of features that yielded highest classification performance ( $A \cup C=0.91$, specificity $=1$, sensitivity $=0.79$, accuracy $=0.84$ ). c) At the same example point, the correlation between the predicted likelihood of seizure relapse and the severity of seizure outcome at year 1 is shown. The predicted likelihood of seizure relapse was significantly different between ILAE 2 and ILAE 3-5 patients combined ( $p=0.003, d=0.95$ ). 


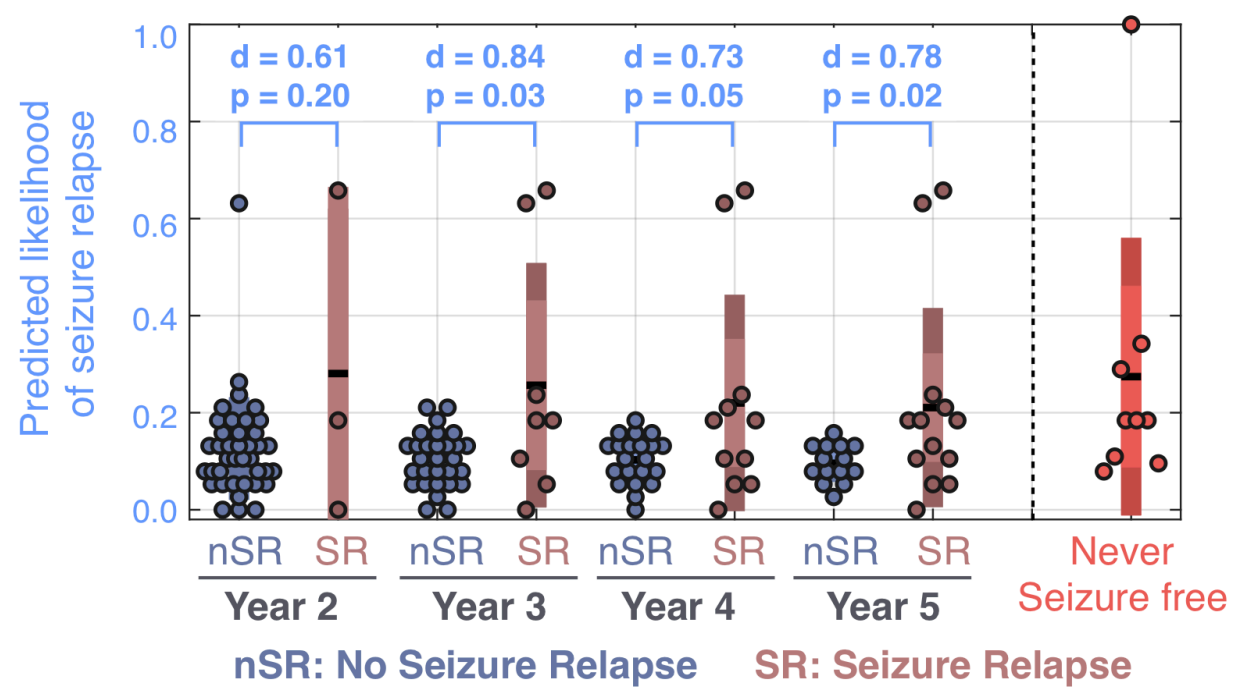

Figure 6: Predicted likelihood of seizure relapse at one-year was higher in patients who had seizure relapse at later years. The predicted 12-month likelihood of seizure relapse was estimated from the SVM model trained with only the surgically-spared node abnormality feature. The likelihood of seizure relapse for patients who were never seizure free (i.e. ILAE 3-6 at year 1 ) are shown in red. Amongst the patients who were initially seizure-free (i.e., ILAE 1 or ILAE 2 at year 1), higher likelihood of seizure relapse was predicted for those who had a subsequent relapse. This is despite the model being blinded to the outcomes at later years. 